\title{
Inorganic characterizations and filler particles morphology of self-adhesive cements
}

\author{
Paulo Henrique Perlatti D'Alpino ${ }^{a, *}$, Rosemary Pereira Araújo ${ }^{a}$, \\ Alejandra Hortencia Miranda González ${ }^{a}$, Vinicius di Hipólito ${ }^{a}$, Claudete Justina Valduga ${ }^{\text {b }}$, \\ Dayse Iara dos Santos ${ }^{c}$, Carlos Frederico Graeff ${ }^{c}$ \\ a Biomaterials Research Group, School of Dentistry, Universidade Anhanguera de São Paulo (UNIAN - SP), São Paulo, SP, Brazil \\ ${ }^{\mathrm{b}}$ Department of Pharmacy and Biotechnology, Universidade Anhanguera de São Paulo (UNIAN - SP), São Paulo, SP, Brazil \\ ${ }^{\mathrm{c}}$ DF-FC, UNESP - Universidade Estadual Paulista, POSMAT - Programa de Pós-Graduação em Ciência e Tecnologia de Materiais, Bauru, SP, Brazil
}

\section{A R T I C L E I N F O}

Article history:

Accepted 1 February 2016

Available online 15 February 2016

Keywords:

Self-adhesive resin cements

Thermal analysis

Phase analysis light scattering

Glass transition temperature

\begin{abstract}
A B S T R A C T
This study evaluated the thermal and morphological filler characteristics of self-adhesive resin cements. The cements (Embrace WetBond, MaxCem Elite, Bifix SE, G-Cem, and RelyX U200) were manipulated according to the manufacturers' instructions. Thermogravimetric analysis and differential thermal analysis were performed to obtain the glass transition temperature $\left(T_{g}\right)$ and weight loss. Specimens were also obtained to characterize the zeta potential, the mean particle size and distribution, and the polydispersity by dynamic light scattering. An elemental analysis of the fillers was also conducted using X-ray spectroscopy analysis and micromorphology under SEM. MaxCem Elite contained the least organic matrix, followed by G-Cem, Bifix SE, RelyX U200, and Embrace WetBond. Bifix SE presented the highest $T_{g}$ and G-Cem the lowest. Bifix SE presented the broadest filler size distribution, exhibiting lower zeta potentials and mobility. G-Cem was found to be a highly filler loaded cement with the lowest effective diameter, highest zeta potential and mobility. RelyX U200 presented chromium in the composition and G-Cem presented fluorine. Differences in the nature and chemistry of inorganic fractions seemed to dictate the morphology of the filler content and also the thermal behavior of the materials tested and, may consequently influence the clinical performance of self-adhesive resin cements.
\end{abstract}

(c) 2016 Elsevier Ltd. All rights reserved.

\section{Introduction}

Filler load and consequential mechanical and physical properties of dental composite materials can be partly related to the nature of the particles [1]. The incorporation of filler particles into a resin matrix and filler characteristics (i.e., radiopacity, filler distribution, shape, and size) changes the physical properties, such as elastic modulus, compressive and tensile strength [2,3]. Thus, the size of the inorganic fractions is a valuable indicator of quality and performance of composite materials [4]. Specifically for luting cements, not only is the relative size and shape distribution of the powder particles important for the viscosity of the cement, but also

\footnotetext{
* Correspondence to: Universidade Anhanguera de São Paulo, UNIAN - SP, Programa de Mestrado em Biomateriais em Odontologia, Av. Raimundo Pereira de Magalhães, 3.305, São Paulo, SP CEP: 05145-200, Brazil. Tel.: +55 1135128400.

E-mail addresses: paulodalpino@yahoo.com (P.H.P. D'Alpino), rosemarycarv@uol.com.br (R.P. Araújo), alejandra.horten@uol.com.br (A.H.M. González),

vdhipolito@yahoo.com.br (V. di Hipólito), cvalduga@usp.br (C.J. Valduga), dayse@fc.unesp.br (D.I. dos Santos), graeff@fc.unesp.br (C.F. Graeff).
}

different packing densities can be achieved with an appropriate selection of the particle format and size [5]. Different parameters such as particle size, viscosity, fillers, and polymerization reactions may also affect the film thickness of resin cements [6]. The rheological properties of the material influence its handling characteristics, for example, the cement must flow readily under pressure to form a thin film [7]. In addition, the amount of fillers and their influence in the decrease of the mobility of polymer radicals have been associated with the decrease of the reactivity of monomers, impacting the polymerization process [8]. In this way, it is suggested that a practical limit to the amount of filler particles in resin cement formulations must be far less than the densest pack limit possible [9].

Resin cements are low-viscosity composites, containing reduced filler content and a resin matrix based on different monomers, such as Bis-GMA, TEGDMA, and methacrylates [10]. Other components like glasses and/or ceramic fillers that contain chemical elements such as barium, strontium, and zirconium can be added to provide radiopacity characteristics [11]. Resin cements have shown improved properties with the incorporation of fillers and also because of the bonding established between fillers and the resin matrix, which is provided by 
a silane coupling agent [2]. It has also been claimed that the fillers play an important role in the self-adhesive resin cements, materials that require no technique-sensitive steps such as acid-etching, priming, and bonding [12], as the initial low acidity of the cement is quickly neutralized during the polymerization process in part due to the chemical interaction of the phosphoric acid groups with the basic inorganic fillers $[13,14]$.

The aim of this study was to investigate and compare thermal characteristics of commercial self-adhesive resin cements. Thermogravimetric analysis and differential thermal analysis were performed to obtain the glass transition temperature $\left(T_{g}\right)$ and weight loss. The inorganic fractions were morphologically characterized using scanning electron microscopy at different magnifications with the respective elemental mapping. Filler morphology was also investigated for their effective diameter, volume, number, zeta potential (colloidal stability), mobility, and polydispersity using dynamic light scattering analysis.

\section{Materials and methods}

\subsection{Experimental design}

In this in vitro study, thermal and morphological characterizations of five commercial self-adhesive resin cements were performed, including Embrace WetBond, MaxCem Elite, Bifix SE, G-Cem, and RelyX U200. The characteristics of the resin cements selected are described in Table 1.

\subsection{Characterization of the self-adhesive resin cements by thermal analysis}

Thermogravimetric analysis (TGA) and differential thermal analysis (DTA) were simultaneously used for thermal characterization of the selected cements. Approximately $60 \mathrm{mg}$ of each SARC, photoactivated according to the manufacturers' instructions, was placed in standard NETZSCH alumina $85 \mu \mathrm{L}$ crucibles attached to the thermoanalytical unit (TGA-50 Netszch-Thermische Analyse, Selb, Germany) with a TA System Controller (TASC 414/2), a temperature range of $50-800{ }^{\circ} \mathrm{C}$, at a heating rate of $10^{\circ} \mathrm{C} / \mathrm{min}$, and under dynamic nitrogen atmosphere $(50 \mathrm{~mL} / \mathrm{min})$. TGA measured the change in mass of the cements as a function of temperature. In DTA, the cement and the inert reference underwent identical thermal cycles, while recording any temperature differences between the specimens and the reference. This differential temperature of the cements was plotted against the heating. Changes in the temperature of the specimens, either exothermic or endothermic, were detected relative to the reference. The baseline to correct the thermo-analytical curves of the specimens was also performed for both analyses using empty alumina crucibles under the same experimental conditions.

The glass temperature $\left(T_{g}\right)$, defined as the temperature at half the height of the step (midpoint temperature, $T_{g \text { midpoint }}$ ) according to the German norm DIN 53765, was also determined by means of extending straight lines along the left-hand and right-hand branches of the heat flow curves. $T_{g}$ was then obtained from the point of intersection of the bisecting lines with the measured curves $[15,16]$.

\subsection{Particle size and polydispersity index measurement by phase laser light scattering}

$10 \mathrm{mg}$ of uncured resin cements was diluted in $1.5 \mathrm{~mL}$ of absolute ethanol (G.R.). The diluted sample was centrifuged at $3.000 \mathrm{rpm}$ ( $\mathrm{g}$ force of $1.000 \mathrm{~g}$ ) for $5 \mathrm{~min}$ and the resulting pellet was suspended in absolute ethanol using the same volume of ethanol and centrifuged again. This process was repeated twice. The ethanol was then discarded and the pellet containing the filler particles was suspended in $1.5 \mathrm{~mL}$ of a $0.001 \mathrm{~mol} \% \mathrm{KCl}$ solution $[17,18]$. A fraction of $10 \mu \mathrm{L}$ of the liquid suspension was deposited in a $3.00 \mathrm{~mL} \mathrm{KCl}$ solution in a $3 \mathrm{~cm}^{3}$ polystyrene cuvette with a path length of $10 \mathrm{~mm}$ and analyzed by dynamic light scattering (DLS, 90 Plus, Brookhaven Instruments Corporation, Holtsville, New York, USA). The filler particles' size distribution (number and volume in $\mathrm{nm}$ ), the effective diameter (in $\mathrm{nm}$ ), and the polydispersity index of each resin cement were then obtained. Ten replications for each resin cement were evaluated $(n=10)$.

Table 1

Self-adhesive resin cements characterized in the present study. ${ }^{\mathrm{a}}$

\begin{tabular}{|c|c|c|c|c|c|c|}
\hline Material & $\begin{array}{l}\text { Lot no./Expiration } \\
\text { date }\end{array}$ & Composition & $\begin{array}{l}\text { Working time } \\
(\mathrm{min})\end{array}$ & $\begin{array}{l}\text { Setting time } \\
(\mathrm{min})\end{array}$ & $\begin{array}{l}\text { Curing } \\
\text { time }(s)\end{array}$ & $\begin{array}{l}\text { Filler content } \\
\mathrm{W}(\%) \mathrm{V}(\%)\end{array}$ \\
\hline Embrace WetBond & 130711 & Co-monomers (mono-, di-, and tri-functional methacrylate & 2 & 3 & 40 & 36.6 \\
\hline $\begin{array}{l}\text { Pulpdent Corporation, } \\
\text { Watertown, MA, USA }\end{array}$ & 2015-07 & $\begin{array}{l}\text { monomers, Barim, glass, ytterbium trifluoride, inert } \\
\text { minerals.) Automix system. }\end{array}$ & & & & 39.0 \\
\hline MaxCem Elite & 5011290 & GPDM, co-monomers (mono-, di-, and tri-functional & 1 & 4 & $10-20$ & 69.9 \\
\hline $\begin{array}{l}\text { Kerr Corporation, Orange, } \\
\text { CA, USA }\end{array}$ & 2015-03 & $\begin{array}{l}\text { methacrylate monomers, water, acetone, and ethanol. Inert } \\
\text { minerals and ytterbium fluoride.) Automix system. }\end{array}$ & & & & 46.0 \\
\hline Bifix SE & 1322421 & Bis-GMA, UDMA, Gly-DMA, phosphate monomers, initia- & 2 & 4 & $10-20$ & 70.0 \\
\hline $\begin{array}{l}\text { Voco GmbH, Cuxhaven. } \\
\text { Germany }\end{array}$ & 2014-12 & tors, stabilizers. Glass. Automix system. & & & & 45.0 \\
\hline G-Cem & 1308051 & Dimethacrylates, 4-META, UDMA, Phosphoric ester mono- & $2^{\prime} 30$ & 4 & 10 & 71.0 \\
\hline $\begin{array}{l}\text { GC Corporation, Tokyo, } \\
\text { Japan }\end{array}$ & 2015-08 & $\begin{array}{l}\text { mer, water; silica powder; Fluoro-alumino-silicate glass } \\
\text { (amorphous), camphorquinone. Capsule. }\end{array}$ & & & & 56.6 \\
\hline RelyX U200 & 1329500659 & $\begin{array}{l}\text { Base: Methacrylate monomers containing phosphoric acid } \\
\text { groups, methacrylate monomers, initiators, stabilizers, } \\
\text { rheological additives. }\end{array}$ & 2 & 6 & 20 & \\
\hline 3M ESPE, & 2014-12 & Catalyst: Methacrylate monomers, alkaline fillers, silanated & & & & 72.0 \\
\hline St. Paul, MN, USA & & $\begin{array}{l}\text { fillers, initiator components, stabilizers, pigments, rheolo- } \\
\text { gical additives. Zirconia/silica fillers. Clicker delivery } \\
\text { system. }\end{array}$ & & & & 43.0 \\
\hline
\end{tabular}

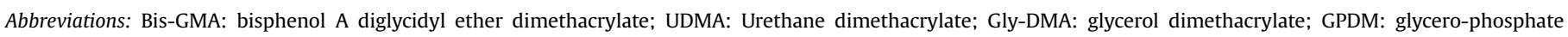
dimethacrylate.

\footnotetext{
${ }^{a}$ Manufacturers's information.
} 


\subsection{Surface zeta potential and mobility measurement by phase analysis light scattering}

The surface charge properties of filler particles dispersed in a potassium chloride $(\mathrm{KCl})$ solution were determined based on the zeta potential measurements using phase analysis light scattering (PALS) (90 Plus, Brookhaven Instruments Corporation, Holtsville, New York, USA). The zeta potential measurement was used to assess the colloidal stability [17] of the fillers in the resin cements according to the experimental groups. The zeta potentials ( $\xi$, in mv) and the mobility $([\mu / \mathrm{s}] /[\mathrm{V} / \mathrm{cm}])$ were calculated from electrophoretic mobility, $\mu$, using the Helmholtz-Smoluchowski Eq. (1) [19]:

$\xi=\mu \eta / \epsilon$

where $\xi$ is the dielectric constant of the medium and $\eta$ is the viscosity of the medium. Ten replications for each resin cement were evaluated $(n=10)$.

\subsection{Characterization of the fillers by SEM imaging observation and energy-dispersive X-ray spectroscopy (EDS)}

In order to evaluate the microstructure of the SARCs, four disks of each material were prepared using Teflon molds $(6 \mathrm{~mm}$ in diameter, $2 \mathrm{~mm}$ thick). After photoactivation, the specimens were immersed in acetone p.a. (99.5\% - Fisher Scientific, Fair Lawn, NY, USA) for $24 \mathrm{~h}$ and then dehydrated in silica gel for $2 \mathrm{~h}$. Next, half of the processed specimens were sputter-coated (40 mA for $120 \mathrm{~s}$ ) with gold/palladium (SCD 050; Balzers, Schaan, Liechtenstein) in order to characterize the inorganic phase of the resin cements by means of SEM (JSM 5600LV - JEOL, Tokyo, Japan) under the secondary electron mode; meanwhile, the other half of the specimens was submitted to carbon evaporation (SCD 050 - Balzers, Schaan, Liechtenstein) for the elemental analysis using EDS under a backscattered electron mode, both operating in high vacuum mode and an accelerating voltage of $20 \mathrm{kV}$. Representative images of selected regions of the sputter-coated specimens were taken in order to characterize the morphological aspect of the filler particles, while a qualitative elemental analysis was performed on the carbon-evaporated ones.

\section{Results}

\subsection{Characterization of the resin cements by thermal analysis}

The simultaneous measurements of thermogravimetric and differential thermal analyses were carried out for all of selected resin cements. Displayed in Fig. 1 are the TGA and DTA curves of the SARCs according to the experimental groups plotted against temperature (in ${ }^{\circ} \mathrm{C}$ ). These analyses revealed comparatively different thermal behavior for each SARC. However, MaxCem Elite, Bifix SE, and G-Cem presented quite similar DTA curves, which can be explained through the following events: the first endothermic range between $30^{\circ} \mathrm{C}$ and $190{ }^{\circ} \mathrm{C}$ can be related to physical desorption and evaporation of water molecules and ethanol. In this range, the TG curves indicated a weight loss of about $1.4 \%, 2.3 \%$, and $1.7 \%$, respectively. The exothermic DTA ranging from 190 to $450{ }^{\circ} \mathrm{C}$ (MaxCem Elite) was accompanied by a weight loss of $23.2 \%$. This same process, observed between $190^{\circ} \mathrm{C}$ and $470{ }^{\circ} \mathrm{C}$ in both Bifix SE and G-Cem, was associated with a weight loss of $29.4 \%$ and $22.3 \%$, respectively. In addition, pronounced endothermic peaks were observed at $638^{\circ} \mathrm{C}$ (MaxCem Elite), $635^{\circ} \mathrm{C}$ (Bifix SE), and $598{ }^{\circ} \mathrm{C}$ (G-Cem). This process involved a small weight loss of $1.9 \%$ from $450^{\circ} \mathrm{C}$ to $685^{\circ} \mathrm{C}$ (MaxCem Elite), and 2.2\%, and 3.6\% (Bifix SE and $\mathrm{G}-\mathrm{Cem}$ ) from $470^{\circ} \mathrm{C}$ to $690^{\circ} \mathrm{C}$. In the sequence, MaxCem Elite had two additional exothermic peaks at both 685 and $755^{\circ} \mathrm{C}$. On
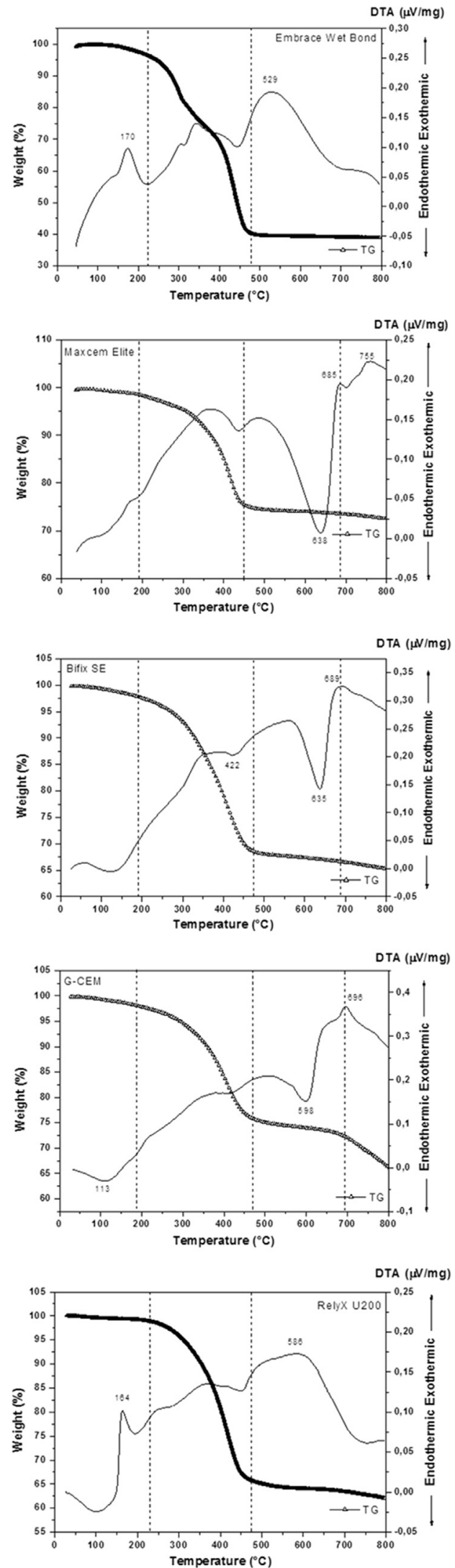

Fig. 1. TG/DTA curves of the self-adhesive resin cements tested.

the other hand, Bifix SE and G-Cem DTA curves exhibited only one additional exothermic peak at $689^{\circ} \mathrm{C}$ and $696^{\circ} \mathrm{C}$, respectively. The final weight loss associated was 1.2\% (MaxCem Elite), 1.3\% (Bifix $\mathrm{SE})$, and $6.1 \%$ (G-Cem). 
Furthermore, Embrace WetBond and RelyX U200 also presented similar DTA curves when compared with each other. The first endothermic band between $30^{\circ} \mathrm{C}$ and $153^{\circ} \mathrm{C}$, observed only in the DTA curve of RelyX U200, can be related to physical desorption and evaporation of water molecules and ethanol. In the first stage, at $164{ }^{\circ} \mathrm{C}$ (for RelyX U200) and at $170{ }^{\circ} \mathrm{C}$ (for Embrace WetBond), the exothermic peaks can be related to the polymerization of unreacted monomers. This initial process involved a small weight loss of $1.0 \%$ from $30^{\circ} \mathrm{C}$ to $230^{\circ} \mathrm{C}$ (RelyX U200) and $3.4 \%$ (Embrace WetBond) from $40^{\circ} \mathrm{C}$ to $221^{\circ} \mathrm{C}$. In the sequence, the exothermic DTA bands for temperatures ranging from 230 to $474{ }^{\circ} \mathrm{C}$ (for RelyX U200) and from $221^{\circ} \mathrm{C}$ and $478{ }^{\circ} \mathrm{C}$ (for Embrace WetBond) were respectively accompanied by a weight loss of $33.1 \%$ and $56.3 \%$. As can be seen in Fig. 1, in this temperature range, the TG curve of Embrace WetBond showed two major changes in weight, indicating different matrix components of the cement. This loss can be possibly related to the removal of residual unreacted monomers, volatile auxiliary materials for the curing reaction, solvents, and water molecules [20]. Finally, both Embrace WetBond and RelyX U200 cements presented a broad exothermic band between $\sim 470{ }^{\circ} \mathrm{C}$ and $800{ }^{\circ} \mathrm{C}$. In the same way, this final exothermic process could be related to the arrangement of new crystalline phases. In this range, the weight loss determined was 3.7\% (for RelyX U200) and 1.5\% (for Embrace WetBond).

In Table 2 the results for the percentage of weight loss are displayed for the different materials studied. In general, the SARCs present according to the manufacturers a concentration of $36.6 \% /$ w (Embrace WetBond) and from 69.9 to $72.0 \% / \mathrm{w}$ for the others (Table 1). The resin cements presented major drop in weight losses of around 250 and $600{ }^{\circ} \mathrm{C}$. The results of thermogravimetric analysis showed an end percentage of weight loss of up to a maximum of $61.2 \%$ (Embrace WetBond). Most of the resin cements showed an end percentage of weight loss from $27.7 \%$ to $37.8 \%$. All the cements demonstrated weight losses of even above $600{ }^{\circ} \mathrm{C}$, which was particularly marked in Embrace WetBond. G-Cem exhibited a shift in the weight loss at higher temperatures $\left(\sim 728^{\circ} \mathrm{C}\right)$. Therefore, the end \% of weight loss for the cement G-Cem was similar to that of Bifix SE, although the former presented a descending curve even at $800{ }^{\circ} \mathrm{C}$. MaxCem Elite exhibited the lowest end percentage of weight loss. Table 2 also presents the glass transition temperatures $\left(T_{g}\right)$ for the different cements. All of the SARCs tested presented a $T_{g}$ in the temperature ranging between $147^{\circ} \mathrm{C}$ and $174{ }^{\circ} \mathrm{C}$. Bifix SE presented the highest $T_{g}$ among cements tested.

\subsection{Filler sizing measurements}

The effective diameter of the fillers and the polydispersity index in the resin cements according to each SARC are also presented in Table 3. Fig. 2 shows the size distribution curves of the fillers as functions of intensity, volume, and number. It was demonstrated that a varied population of larger and smaller particles coexisted in the resin cements (Fig. 2). The cement Bifix SE presented higher effective diameter and higher variability in terms of filler size distribution compared to other SARCs. After calculating the difference between the maximum and the minimum particle sizes present in

Table 2

Percentage of mass loss and glass transition temperature of self-adhesive resin cements tested.

\begin{tabular}{llll}
\hline Resin cement & \% Weight loss & Filler load (\%) & $\boldsymbol{T}_{\mathbf{g}}\left({ }^{\circ} \mathbf{C}\right)$ \\
\hline Embrace WetBond & 61.2 & 39.4 & 162 \\
MaxCem Elite & 27.7 & 72.3 & 156 \\
Bifix SE & 35.2 & 64.8 & 174 \\
G-Cem & 33.7 & 66.3 & 147 \\
RelyX U200 & 37.8 & 62.2 & 154 \\
\hline
\end{tabular}

the resin cements, it was possible to note that Bifix SE showed the broadest filler size distribution, followed by Embrace WetBond, MaxCem Elite, RelyX U200, and G-Cem (Table 3).

The filler size distribution in terms of number and volume was also displayed in Table 3 and Fig. 2. It was demonstrated that MaxCem Elite presented the broadest and G-Cem the narrowest filler size distribution, in terms of mean and median filler number. On the other hand, in terms of mean and median volume, Bifix SE presented the broadest filler distribution and G-Cem, the narrowest. All of the resin cements presented a mid-range polydispersity (0.08-0.7).

\subsection{Zeta potential}

Table 3 also displays the variation of $\xi$ potential and the mobility observed in the SARCs. The $\xi$ potentials of fillers in the potassium chloride solution were positive over the entire range of measurement, which indicates positive surface charge in the solution. The $\xi$ potential in the cement G-Cem was more positive $(46.4 \mathrm{mV})$ and Bifix SE, less $(16.4 \mathrm{mV})$. The other cements presented intermediary averages. MaxCem Elite and RelyX U200 exhibited similar $\xi$ potentials ( 34.1 and $33.7 \mathrm{mV}$, respectively). These results indicate that the dispersion of particles in G-Cem was more stable in a solution. In addition, the mobility in Bifix SE was comparatively lower than that observed in the other cements whereas in G-Cem, it was higher.

\subsection{SEM/EDS analysis}

Representative SEM/EDS analyses are presented in Fig. 3 (from A to $E$ ). The illustrations revealed that there was great variability in terms of shape and size of the fillers present in the SARCs tested. The illustrations revealed that MaxCem Elite and RelyX U200 contained filler particles that were sparsely distributed, characteristics of a non-uniform particle size distribution. In both resin cements, there were larger fillers with the size in some cases varying from 10 to $15 \mu \mathrm{m}$. The illustrations also demonstrated GCem had lower sized fillers with a more uniform distribution in terms of size and shape.

The EDS analysis revealed a higher incidence of Si and a considerable amount of $\mathrm{Al}$ in the composition of all of the SARCs tested. This may be related to the presence of silica $\left(\mathrm{SiO}_{2}\right)$ and alumina $\left(\mathrm{Al}_{2} \mathrm{O}_{3}\right)$ in the composition. A considerable amount of titanium was observed in Embrace WetBond and MaxCem Elite, related to the presence of Titania fillers $\left(\mathrm{TiO}_{2}\right)$. G-Cem presented a relatively higher concentration of fluoride ions, not observed in the other SARCs. Bifix SE showed varied peaks of barium, possibly related to the presence of glass filler particles of barium glass. RelyX U200 also presented a relatively lower concentration of chromium in the elemental mapping. Other ions were also found in lower concentrations as observed in Fig. 3.

\section{Discussion}

Thermogravimetric analysis of resin cements provides important information regarding the structure and the influence of the organic matrix [21]. It has been also claimed that the filler content can be estimated from the weight remaining after the last observed weight change at about $575{ }^{\circ} \mathrm{C}$ [22], according to the ISO 4049. The glass transition temperature $T_{g}$ is an important physical characteristic that determines the physical state of a polymer and influences several properties [23]. On heating to temperatures above $T_{g}$, the polymers become elastic like rubber or liquefy depending on their molecular weight and/or degree of crosslinking [21]. Crosslinking chemically links molecules, while 
Table 3

Summary of the results of the laser light scattering analysis and phase analysis light scattering of the self-adhesive resin cements tested

\begin{tabular}{|c|c|c|c|c|c|c|c|c|}
\hline \multirow[t]{2}{*}{ Composite } & \multirow[t]{2}{*}{ Effective Diameter (nm) } & \multicolumn{2}{|c|}{ Number distribution } & \multicolumn{2}{|c|}{ Volume distribution } & \multirow[t]{2}{*}{ Polydispersity } & \multirow[t]{2}{*}{ Zeta potential (mv) } & \multirow[t]{2}{*}{ Mobility $(\mu / \mathrm{s}) /(\mathrm{V} / \mathrm{cm})$} \\
\hline & & Mean (nm) & Median (nm) & Mean (nm) & Median (nm) & & & \\
\hline Embrace WetBond & $3661(628)$ & $799(382)$ & 719 & $1884(900)$ & 1696 & $0.4(0.1)$ & $22.0(4.1)$ & $1.7(0.3)$ \\
\hline MaxCem Elite & $3520(515)$ & 1136 (469) & 1049 & $2158(891)$ & 1994 & $0.3(0.1)$ & $34.1(5.5)$ & $2.7(0.4)$ \\
\hline Bifix SE & 3852 (909) & $898(440)$ & 804 & 2218 (1089) & 1985 & $0.4(0.1)$ & $16.4(5.0)$ & $1.3(0.4)$ \\
\hline G-Cem & $1477(117)$ & $549(211)$ & 513 & $959(368)$ & 895 & $0.3(0.1)$ & $46.4(4.6)$ & $3.6(0.4)$ \\
\hline RelyX U200 & $2465(130)$ & $743(318)$ & 683 & $1481(634)$ & 1360 & $0.3(0.1)$ & $33.7(6.8)$ & $2.6(0.5)$ \\
\hline
\end{tabular}

In parenthesis - standard deviation.

crystallinity and fillers introduce physical limitations to monomer mobility [24]. The use of monomers that form a rigid polymer network with higher cross-linking density can lead to a resinbased material with a high $T_{g}$ [21].

TGA characterization revealed different thermal behavior when the results were compared. As the temperature increased, resin cements degraded losing weight. In this process, a series of decomposition reactions may occur: vaporization (physical), desorption (physical), oxidation (physical), decomposition (chemical), dehydration, and desolvation (chemical) [25]. The DTA curves showed that the monomeric phase of the resin cements decomposed when heated over $300{ }^{\circ} \mathrm{C}$. Excepted for Embrace WetBond, all SARCs exhibited a decomposition at higher temperature (peak at $\sim 400{ }^{\circ} \mathrm{C}$ ), characteristic of the decomposition of BisGMA-based restorative materials [25]. Embrace WetBond exhibited a different thermal behavior, with varied exothermic peaks. The first main decomposition peak seen for Embrace WetBond was found at $\sim 437^{\circ} \mathrm{C}$. Differences in the chemical structures of pure and resin materials based on Bis-GMA, Bis-EMA, UDMA, and TEGDMA considerably influence their degradation behavior according to their thermal degradation mechanisms [26]. Pure Bis-GMA and Bis-EMA monomers exhibit one-step degradation mechanisms with a $T_{\text {peak }}$ at 415 and $424^{\circ} \mathrm{C}$ respectively, whereas TEGDMA and UDMA present two degradation steps with a $T_{\text {peak }}$ at $306{ }^{\circ} \mathrm{C}$ and $403{ }^{\circ} \mathrm{C}$ for TEGDMA and $357^{\circ} \mathrm{C}$ and $444^{\circ} \mathrm{C}$ for UDMA [27].

In the present study, except for MaxCem Elite, all of the resin cements showed a weight loss higher than that of the organic matrix content informed by the manufacturer after heating the resin cements up to a temperature of $800{ }^{\circ} \mathrm{C}$. Therefore, the results indicated that the SARC MaxCem Elite contained the least organic matrix compared to G-Cem, Bifix SE, RelyX U200, and Embrace WetBond, sequentially (Table 2). The highest $T_{g}$ was for Bifix SE $\left(174{ }^{\circ} \mathrm{C}\right)$, followed by Embrace WetBond, MaxCem Elite, RelyX U200, and G-Cem. $T_{g}$ variation can be attributed to various molecular parameters, such as molecular weight, stiffness of the cross-linked chains, and free volume entrapped in the network [28]. Other studies have shown that $T_{g}$ can be affected by cross-linked chains and filler content [29].

Zeta potential is an important physical property exhibited by particles when in suspension [30]. The magnitude of the zeta potential gives an indication of the potential stability of a colloidal system [31]. Most colloidal dispersions in aqueous media carry an electric charge. The origin of surface charges varies depending on the nature of the particle and its surrounding medium. If the fillers in suspension have a large negative or positive zeta potential, a tendency to repel each other exists and there is a lower tendency for the fillers to aggregate. If the particles have low zeta potential, or are electrically neutral, no forces may exist to prevent the particles to flocculate and aggregate [32].

Manufacturers have presented in the technical product profiles an estimation of the filler size found in the resin-based materials. In addition, for some products this information is either unclear or not provided in the technical profile. Concerns have been expressed as researchers have drawn their conclusions based on this information alone. In general, it has been claimed that the resin cement composition is generally similar to that of resin composites with the difference in terms of volumetric percentage of fillers, which is lower in the former due to an adequate viscosity needed for a luting cement material [11]. In this way, the characterization of fillers in terms of size, volume, shape, and packing is of paramount importance to understanding the findings. Although it was demonstrated that filler distribution by weight varied among the cements, the results of laser dynamic light scattering method showed a broad distribution of fillers in terms of effective diameter, number, and volume. For example, G-Cem is a cement with a high load of filler (66.3\%/weight) but the fillers presented the lowest effective diameter $(1.4 \mu \mathrm{m})$, number $(\sim 550 \mathrm{~nm})$, and volume $(\sim 960 \mathrm{~nm})$ distribution in comparison to other cements. Conversely, Bifix SE is a highly filler-loaded cement as well (64.8\%/weight) but comparatively presented the highest effective diameter $(3.9 \mu \mathrm{m})$ and the highest volume distribution $(2.2 \mu \mathrm{m})$. The distribution in terms of number was intermediary $(\sim 800 \mathrm{~nm})$ in comparison to that of other SARCs. The topographic SEM study showed a great variability in terms of shape, size, and concentration of fillers present in the SARCs tested (Fig. 3). In this case, the SEM analysis clearly indicates that MaxCem Elite, G-Cem, and RelyX U200 present characteristics of a non-uniform particle size distribution, containing filler particles that are larger and sparsely distributed compared to Embrace WetBond and Bifix SE. Moreover, these representative figures demonstrate that the fillers are irregular in shape and size.

Disagreements in terms of volume and shape occur when the fillers are measured using different methods [33]. It has been generally recommended to use more than one technique to measure the diameter of fillers. TEM and SEM provide only core measurements, because the coating is not normally imaged by the electrons. Also, when having aggregates, it can be difficult to identify distinct fillers [34]. Conversely, the laser light scattering method averages the various dimensions as the particles flow randomly through the light beam, producing a distribution of sizes from the smallest to the largest dimensions [33]. In spite of this advantage, the range at which this method detects the filler size distribution varies from $0.3 \mathrm{~nm}$ to $6 \mu \mathrm{m}$ (manufacturer's information). This explains the difference in terms of filler size distribution between the SEM and laser light scattering method.

The stability of the inorganic fractions in the resin cements is influenced by steric and electrostatic stabilization that induce particle repulsion, whereas kinetic stability can be induced by increasing the viscosity of the suspending medium, thus slowing down particle aggregation and sedimentation [35]. Controlling the dispersion of fillers is very difficult, considering the highly filled, viscous systems such as those of resin cements [1]. Although the filler size distribution in terms of intensity represents a direct measurement and seems to be the most accurate approach, the presence of bigger filler sizes in the specimens may be possibly overestimated. This can be explained by the fact that bigger particles scatter more light and also occupy more space, even if they 

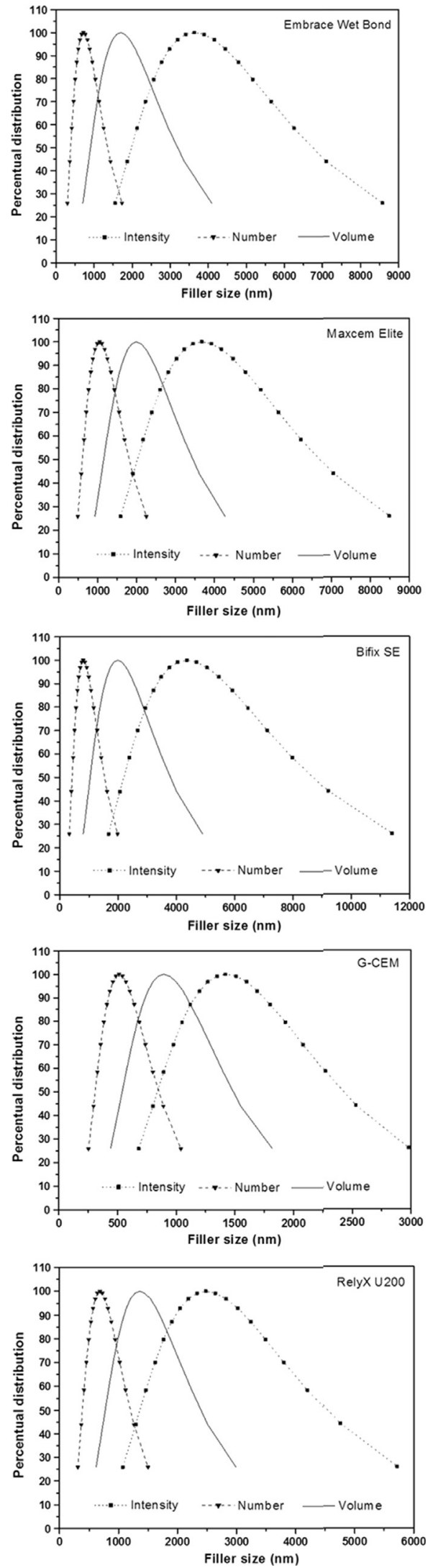

Fig. 2. Particle intensity, number, and volume distribution curves of the fillers in the resin cements tested.

are the minority in the specimen [36]. On the other hand, volume distribution distinguishes the different morphologies in a multiphase mixture of particles providing a better morphology distribution of the fillers. In this case, each type of filler morphology has its own accumulative peak. In the case of the presence of lower amount of larger filler particles in a specimen, the intensity and volume will tend to represent a superior diameter, because those bigger filler sizes scatter more light and also occupy more space [37]. In number distribution, however, less weight is represented in the distribution because they are the minority. In this way, the filler size distribution based on numbers is not usually reported, unless in the case where a narrow range of fillers exists, which is not the case of all of the resin cements tested. This is the reason why intensity data (which is usually between the volume and size distribution) is normally provided. In the present study, in order to understand how prevalent species and how relative abundance of population components are, filler distribution in terms of intensity, number, and volume was provided.

As previously discussed, the filler distribution varied among the resin cements tested, irrespective of the method employed as observed in Fig. 3. In the present study, the zeta potential of Bifix SE was the least positive and G-Cem, the most. As previously explained, a tendency to the neutralization of the zeta potential may induce the filler aggregation. On the other hand, for MaxCem Elite and RelyX U200, similarities in the zeta potential were also found. In addition, there was found a very strong negative correlation $\left(R^{2}=-0.75\right)$ between the effective diameter and mobility considering the results of the resin cements (data not shown). This correlation is particularly important considering the rheological characteristics of resin cements needed when bonding indirect restorations.

Manufacturers have also presented in their technical product profiles the natures and the chemical compositions of the fillers added to the resin cements. The elements detected were silica ( $\mathrm{Si})$, aluminum (Al), magnesium (Mg), fluorine (F), titanium (Ti), oxygen $(\mathrm{O})$, chromium $(\mathrm{Cr})$, strontium $(\mathrm{Sr})$, tungsten $(\mathrm{W})$, zinc $(\mathrm{Zn})$, phosphorus $(\mathrm{P})$, sodium $(\mathrm{Na})$, and barium $(\mathrm{Ba})$. The EDS analysis also revealed predominance of $\mathrm{Si}$ in all of the SARCs (Fig. 3) due to the presence of silica $\left(\mathrm{SiO}_{2}\right)$ in the composition of the resin cements. There were also noted lower concentrations of titanium ion in Embrace WetBond and MaxCem Elite, related to the presence of Titania $\left(\mathrm{TiO}_{2}\right)$ in their composition. Bifix SE showed peaks of barium, possibly related to the presence of barium glass fillers and/or barium glass aluminum. Fluoride ion was only found in a detectable amount in the SARC G-Cem. Other ions were also detected in minor concentrations. Another important finding was the presence of chromium ion in the formulation of RelyX U200, known for its cytotoxicity, to cause immunologic problems, and also for being mutagenic in certain species [38].

Clinicians may select commercial resin cements expecting enhanced physical properties. Considering that the decision can be made based on the manufacturer's reporting of the organic components and also on the filler content, the clinician needs to be aware of the importance of both components $[39,40]$. The use of different and complementary methods to characterize the inorganic fractions of the self-adhesive resin cements in the present study demonstrated that the filler weight percentage varied among the cements tested. The filler load and consequential mechanical and physical properties are partly related to the nature of the particles [1]. The differences in terms of the nature and chemistry of the inorganic fractions found in the materials seem to dictate the size and shapes of the filler content and also the thermal behavior and, may consequently influence the clinical performance of self-adhesive resin cements. Extrapolations to clinically support the choice of different SARCs tested need to be done with caution since future studies are necessary in order to complement these results. 

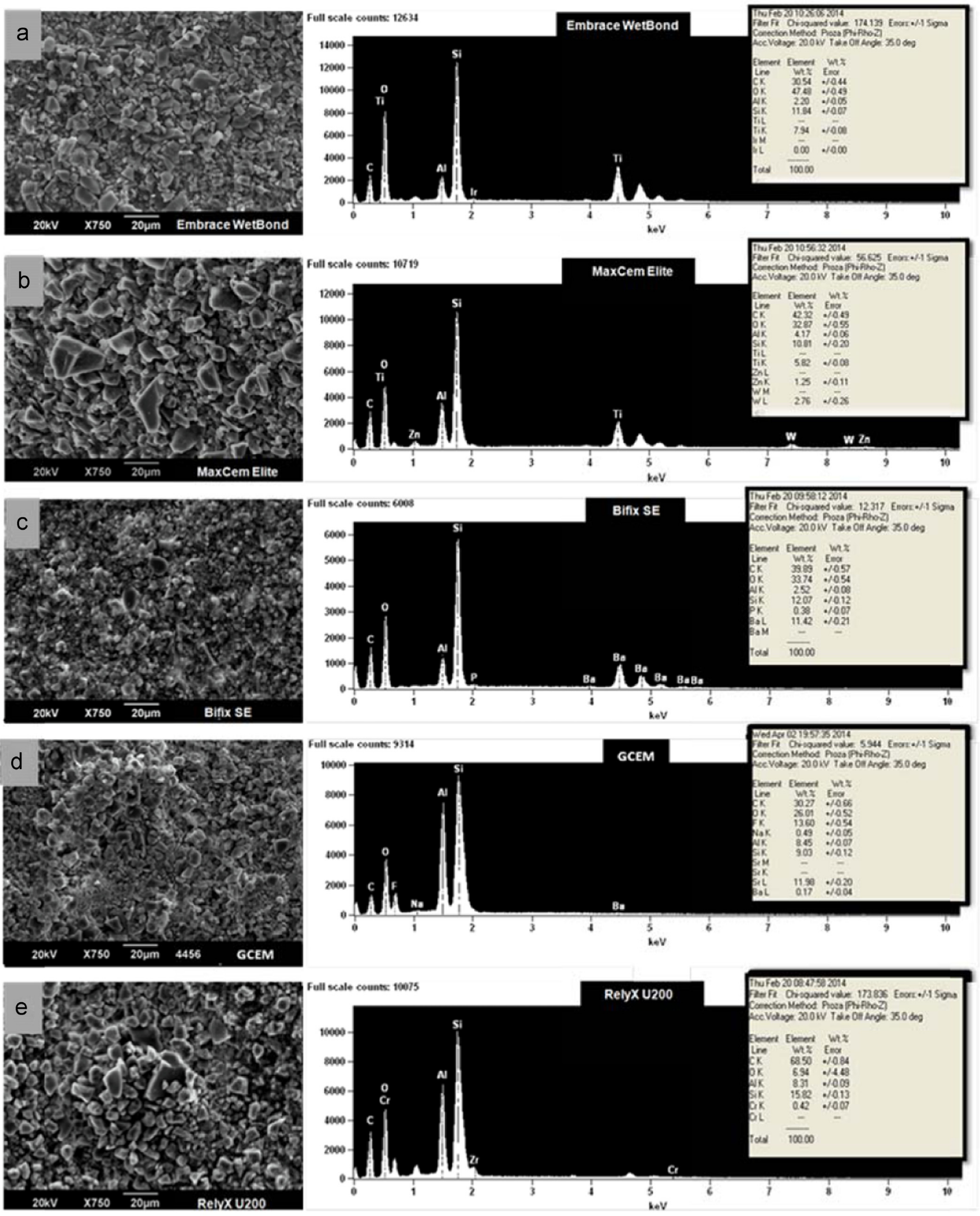

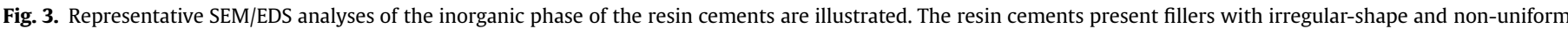

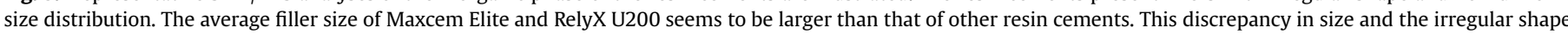

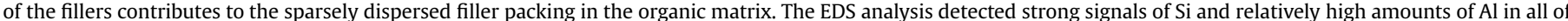

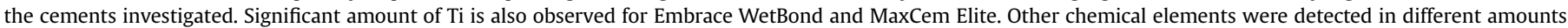

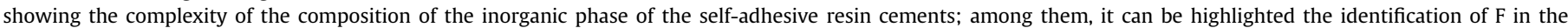
composition of G-Cem taking in account its anti-cariogenic activity, and $\mathrm{Cr}$ in RelyX U200 due to its mutagenic potential. 


\section{Conclusions}

The thermal behavior varied among the self-adhesive resin cements tested. MaxCem Elite contained the smallest organic matrix content, followed by G-Cem, Bifix SE, RelyX U200, and Embrace WetBond. Bifix SE presented the highest $T_{g}\left(174^{\circ} \mathrm{C}\right)$ and $\mathrm{G}$-Cem, the lowest $\left(147^{\circ} \mathrm{C}\right)$. Bifix SE also presented the broadest filler size distribution in comparison to other cements, exhibiting lower zeta potentials and mobility. G-Cem was found to be highly filler-loaded cement with conversely lower effective diameter and filler size distribution in comparison to other cements. G-Cem also presented the highest zeta potential and mobility. The elements detected were silica $(\mathrm{Si})$, aluminum $(\mathrm{Al})$, magnesium $(\mathrm{Mg})$, fluorine $(\mathrm{F})$, titanium (Ti), oxygen $(\mathrm{O})$, chromium $(\mathrm{Cr})$, strontium $(\mathrm{Sr})$, tungsten $(\mathrm{W})$, zinc $(\mathrm{Zn})$, phosphorus $(\mathrm{P})$, sodium $(\mathrm{Na})$, and barium (Ba). $\mathrm{Si}, \mathrm{Al}$, and $\mathrm{O}$ were the common elements in the composition of all five self-adhesive resin cements evaluated.

\section{Acknowledgments}

This study was developed as partial fulfillment of the requirements of Dr. Araújo's Master degree (UNIAN - SP), Brazil. The authors are also grateful to Prof. Dr. Francisco André Ossamu Tanaka from NAP/MEPA (Support Research Nucleus/Electron Microscopy Applied to Agriculture - ESALQ/USP) - Piracicaba, for the support in the SEM analysis.

\section{References}

[1] Leprince J, Palin WM, Mullier T, Devaux J, Vreven J, Leloup G. Investigating filler morphology and mechanical properties of new low-shrinkage resin composite types. J Oral Rehabil 2010;37:364-76.

[2] Polydorou O, Konig A, Hellwig E, Kummerer K. Long-term release of monomers from modern dental-composite materials. Eur J Oral Sci 2009;117:68-75.

[3] Kim KH, Ong JL, Okuno O. The effect of filler loading and morphology on the mechanical properties of contemporary composites. J Prosthet Dent 2002;87:642-9.

[4] Beun S, Glorieux T, Devaux J, Vreven J, Leloup G. Characterization of nanofilled compared to universal and microfilled composites. Dent Mater 2007;23:51-9.

[5] Svizero NR, Silva MS, Alonso RC, Rodrigues FP, Hipolito VD, Carvalho RM, et al Effects of curing protocols on fluid kinetics and hardness of resin cements. Dent Mater J 2013;32:32-41.

[6] Nakamura T, Wakabayashi K, Kinuta S, Nishida H, Miyamae M, Yatani H. Mechanical properties of new self-adhesive resin-based cement. J Prosthodont Res 2010:54:59-64.

[7] Di Hipolito V, Rodrigues FP, Piveta FB, Azevedo Lda C, Bruschi Alonso RC Silikas N, et al. Effectiveness of self-adhesive luting cements in bonding to chlorhexidine-treated dentin. Dent Mater 2012;28:495-501.

[8] Pereira SG, Fulgencio R, Nunes TG, Toledano M, Osorio R, Carvalho RM. Effect of curing protocol on the polymerization of dual-cured resin cements. Dent Mater 2010;26:710-8.

[9] Darwell B. Resin restorative materials. In: Materials science for dentistry. Woodhead Publishing Ltd.; 2009. p. 115-45.

[10] Weiner RS. Dental cements: a review and update. Gen Dent 2007;55:357-64 quiz 65-6, 75-6.

[11] Anusavice KJ, Phillips RW. Phillips' science of dental materials. 11th ed. Philadelphia: Elsevier; 2003.

[12] De Munck J, Vargas M, Van Landuyt K, Hikita K, Lambrechts P, Van Meerbeek B. Bonding of an auto-adhesive luting material to enamel and dentin. Dent Mater 2004;20:963-71.

[13] Radovic I, Monticelli F, Goracci C, Vulicevic ZR, Ferrari M. Self-adhesive resin cements: a literature review. J Adhes Dent 2008;10:251-8.
[14] Di Hipólito V, Azevedo LD, Piveta FB, Vieira WS, Anauate-Netto C, Alonso RC, et al. Effect of dentinal surface preparation on the bonding of self-adhesive luting cements. J Adhes Sci Technol 2014;28:1901-24.

[15] Rieger J. The glass transition temperature $T_{\mathrm{g}}$ of polymers-comparison of the values from differential thermal analysis (DTA, DSC) and dynamic mechanical measurements (torsion pendulum). Polym Test 2001;20:199-204.

[16] Rieger J. The glass transition temperature of polystyrene. J Therm Anal 1996;46:965-72.

[17] Knowles JC, Callcut S, Georgiou G. Characterisation of the rheological properties and zeta potential of a range of hydroxyapatite powders. Biomaterials 2000;21:1387-92.

[18] D'Alpino PH, Svizero ND, Bim Junior O, Valduga CJ, Graeff CF, Sauro S. Effects of age condition on the distribution and integrity of inorganic fillers in dental resin composites. Clin Oral Investig 2015.

[19] Zha LS, Li L, Bao LY. Synthesis and colloidal stability of poly(N-isopropylacrylamide) microgels with different ionic groups on their surfaces. J Appl Polym Sci 2007;103:3893-8.

[20] Soanca A, Roman A, Moldovan M, Perhaita I, Tudoran L, Rominu M. Study on thermal behaviour, structure and filler morphology of some indirect composite resins. Dig J Nanomater Biostruct 2012;7:1071-81.

[21] D'Alpino PHP, Vismara MVG, Mello LMdM, Hipólito VD, González AHM, Graeff CFdO. Resin composite characterizations following a simplified protocol of accelerated aging as a function of the expiration date. J Mech Beh Biom Mater 2014:35:59-69.

[22] Sabbagh J, Ryelandt L, Bacherius L, Biebuyck JJ, Vreven J, Lambrechts P, et al. Characterization of the inorganic fraction of resin composites. J Oral Rehabil 2004;31:1090-101.

[23] Boyer RF. Encyclopedia of Polymer Science and Technology. New York: Wiley Interscience; 1977

[24] Charton C, Falk V, Marchal P, Pla F, Colon P. Influence of $T_{\mathrm{g}}$, viscosity and chemical structure of monomers on shrinkage stress in light-cured dimethacrylate-based dental resins. Dent Mater 2007;23:1447-59.

[25] Vaidyanathan J, Vaidyanathan TK, Wang Y, Viswanadhan T. Thermoanalytical characterization of visible light cure dental composites. J Oral Rehabil 1992;19:49-64.

[26] Sideridou ID, Karabela MM, Vouvoudi E. Physical properties of current dental nanohybrid and nanofill light-cured resin composites. Dent Mater 2011;27:598-607.

[27] Sideridou I, Achilias DS, Kyrikou E. Thermal expansion characteristics of lightcured dental resins and resin composites. Biomaterials 2004;25:3087-97.

[28] Min BG, Stachurski ZH, Hodgkin JH. Cure kinetics of elementary reactions of a DGEBA/DDS epoxy resin: 1. Glass transition temperature versus conversion. Polymer 1993;34:4908-12.

[29] Emami N, Soderholm KJ. Dynamic mechanical thermal analysis of two lightcured dental composites. Dent Mater 2005;21:977-83.

[30] Du M, Zheng Y. Modification of silica nanoparticles and their application in udma dental polymeric composites. Polym Compos 2007;28:198-207.

[31] Sadat-Shojai M, Atai M, Nodehi A, Khanlar LN. Hydroxyapatite nanorods as novel fillers for improving the properties of dental adhesives: synthesis and application. Dent Mater 2010;26:471-82.

[32] Thiemig D, Bund A. Influence of ethanol on the electrodeposition of $\mathrm{Ni} / \mathrm{Al}_{2} \mathrm{O}_{3}$ nanocomposite films. Appl Surf Sci 2009;255:4164-70.

[33] Brown SC, Palazuelos M, Powers KW, Roberts SM, Grobmyer SR, Moudgil BM. Nanoparticle characterization for cancer nanotechnology and other biological applications. In: Grobmyer SR, Moudgil BM, editors. Cancer nanotechnology methods and protocols. London: Springer; 2010. p. 39-65.

[34] Salazar DC, Dennison J, Yaman P. Inorganic and prepolymerized filler analysis of four resin composites. Oper Dent 2013;38:E201-9.

[35] Larsson M, Hill A, Duffy J. Suspension stability; why particle size, zeta potential and rheology are important. Annu Trans Nord Rheol Soc 2012;20:209-14.

[36] Lee Black D, McQuay MO Bonin MP. Laser-based techniques for particle-size measurement: a review of sizing methods and their industrial applications. Prog Energy Combust Sci 1996;22:267-306.

[37] Weiss EL, Frock HN. Rapid analysis of particle size distributions by laser light scattering. Powder Technol 1976;14:287-93.

[38] Black J. Does corrosion matter? J Bone Jt Surg Br 1988;70:517-20.

[39] D'Alpino PH, Silva MS, Vismara MV, Di Hipolito V, Miranda Gonzalez AH, de Oliveira Graeff CF. The effect of polymerization mode on monomer conversion, free radical entrapment, and interaction with hydroxyapatite of commercial self-adhesive cements. J Mech Behav Biomed Mater 2015;46:83-92.

[40] Vaz RR, V.D. Hipolito, P.H. D'Alpino, M.F. Goes. Bond strength and interfacial micromorphology of etch-and-rinse and self-adhesive resin cements to dentin. J Prosthodont 2012;21:101-11. 